\title{
What is the Proportion of Patients With Multiple Hereditary Exostoses Who Undergo Malignant Degeneration?
}

\author{
Cory M. Czajka MD, Matthew R. DiCaprio MD
}

Received: 15 September 2014/ Accepted: 5 January 2015/Published online: 13 January 2015

(C) The Association of Bone and Joint Surgeons (B) 2015

\begin{abstract}
Background Multiple hereditary exostoses is an autosomal-dominant skeletal disorder that has a wide-ranging reported risk of malignant degeneration to chondrosarcoma. Questions/purposes The aims of our study were to use a large, web-based survey approach to characterize (1) the demographic distribution of patients with multiple hereditary exostoses, (2) the number of surgeries performed related to one's diagnosis of multiple hereditary exostoses, and (3) the proportion of survey respondents who described experiencing malignant degeneration in a large international, heterogeneous cohort of patients with multiple hereditary exostoses.

Methods An anonymous web-based survey was distributed to several online support groups and social media networks designed to support and educate patients with multiple hereditary exostoses and their families. The survey collected demographic and epidemiologic data on 779 respondents. Data were recorded to assess respondents' disease burden and the rate of malignant degeneration.
\end{abstract}

Each author certifies that he or she has no commercial associations (eg, consultancies, stock ownership, equity interest, patent/licensing arrangements, etc) that might pose a conflict of interest in connection with the submitted article.

All ICMJE Conflict of Interest Forms for authors and Clinical Orthopaedics and Related Research editors and board members are on file with the publication and can be viewed on request.

Each author certifies that his or her institution approved the human protocol for this investigation and that all investigations were conducted in conformity with ethical principles of research.

C. M. Czajka ( $₫)$, M. R. DiCaprio

Division of Orthopaedic Surgery, Albany Medical Center,

98 Middlesex Court, Slingerlands, NY 12159, USA

e-mail: coryczajka@gmail.com
Results Females represented a slightly greater proportion of those with multiple hereditary exostoses who responded (56\% female; 419 of 742 patients). Median age for all respondents was 28 years (range, $<1-85$ years). Median age for males was 25 years (range, $<1-85$ years), while median age for females was 29 years (range, $<1-$ 82 years). The mean age at diagnosis of male and female respondents was in the mid-first decade (5.4 years \pm 7.2 years). The mean number of surgeries a patient had undergone was 7.3 ( \pm 7.1 surgeries). The proportion of respondents who experienced malignant transformation was $2.7 \%$ ( 21 of 757 respondents), at a mean age of 28.6 years ( \pm 9.3 years). The most common sites of malignant change from benign exostoses included the pelvis (eight of 21 respondents) and scapula (four of 21 respondents).

Conclusions In the largest and most geographically diverse study of patients with multiple hereditary exostoses of which we are aware, we found the proportion of patients with multiple hereditary exostoses who have undergone malignant degeneration to be consistent with those reported in prior studies. Our study perhaps more accurately assessed the proportion of patients who undergo malignant transformation of multiple hereditary exostoses. As with prior studies on this topic, the proportion of malignant change may be expected to represent a high-end estimate as recruitment and selection bias likely predisposes for patients with more severe disease, whereas patients with lesser disease may be unaware of their diagnosis. In discussing the sequelae of multiple hereditary exostoses, clinicians perhaps might use this study to offer an unspecific statement of risk of malignant degeneration of multiple hereditary exostoses among the population at large.

Level of Evidence Level IV, prognostic study. 


\section{Introduction}

Multiple hereditary exostoses, also known as multiple osteochondromatosis, is a relatively rare autosomal-dominant skeletal disorder that affects approximately one in 50,000 individuals [13]. The condition is a genetically heterogeneous disorder that has been linked to mutations of the genes EXT1 [2] and EXT2 [15, 16], with a reported penetrance of $96 \%$ [9].

Bony exostoses, which are the hallmark of multiple hereditary exostoses, have a widely variable clinical presentation. Although they can appear at any location associated with a physis, the lesions typically occur at the arm, forearm, thigh, and leg. They also commonly are found about the pelvis and shoulder [7, 9]. Patients often report problems resulting from compression or irritation of local tissues, deformation of joints, or limb-length discrepancies. Complications of bony exostoses include compression of adjacent structures, such as nerves, blood vessels, and other soft tissues, leading to pain and sensory or motor deficits [5, 6]. These symptomatic lesions often require surgical treatment. We propose that a somewhat rudimentary clinical measurement of disease burden entails the number of surgeries one must undergo to treat symptomatic lesions. As such, part of our study aim was to record the number of surgeries patients with multiple hereditary exostoses have undergone as an approximation of their disease burden. There is large variation among patients in the number and location of lesions, and the degree of orthopaedic deformities and how functional impairments manifest [11].

A more serious complication of multiple hereditary exostoses is the malignant transformation of an exostosis to chondrosarcoma and, rarely, to other malignancies [14]. Previous studies have shown a wide range in the numbers of patients with multiple hereditary exostoses who have had malignant change to chondrosarcoma, and these studies [3, 7 , $8,12-14]$ cite proportions derived from smaller population clusters in seemingly isolated geographic areas. Historically, reported proportions of transformation have ranged from less than $1 \%$ to approximately $25 \%$ [3, 4, 7-9, 11-14]. Some of the larger studies we are aware of include rates of $4.9 \%$ in 529 patients [11], $0.38 \%$ in 526 patients [8], and $7 \%$ in 184 patients [4]. Pedrini et al. [11] presented the largest cohort to date in their genotype-phenotype correlation study of 529 patients with multiple hereditary exostoses. They primarily sought to identify "protective" and "risk" factors associated with the disease. Their comprehensive analytic study secondarily identified a malignant progression rate of $4.9 \%$ (26 cases of chondrosarcoma in 529 patients with multiple hereditary exostoses) [11]. Therefore, we sought to determine a more accurate proportion of patients with multiple hereditary exostoses who have had malignant degeneration.
Previous studies have been limited to confined geographic areas. We sought to expand the catchment area by using webbased resources that may have been unavailable to previous researchers on this topic. In using these web-based platforms a truly global cohort may be recruited, thereby perhaps improving the epidemiologic data collected.

\section{Materials and Methods}

After obtaining institutional review board approval, we used a commercially available web-based survey tool (surveymonkey.com) to collect anonymous responses to our questionnaire. The survey was distributed as a web link to multiple online support groups and social media sites dedicated to education of multiple hereditary exostoses and networking for comprehensive care. The sites included www.mheandme.com ("The Bumpy Bone Club"), several Facebook pages dedicated to multiple hereditary exostoses education and online support, and their associated Twitter feeds. The survey also was distributed directly via e-mail to patients with multiple hereditary exostoses in our practice. The support groups and social media sites have memberships consisting not only of patients with multiple hereditary exostoses, but also patients' families, healthcare professionals, and other individuals with an interest in multiple hereditary exostoses education and support. It is unknown how many individuals use the various sites. The site administrators estimate that nearly 3000 unique visitors worldwide cumulatively visit the sites during a typical quarterly period.

With the assistance of the site administrators, our survey link was posted for 3 months in 2013. Before posting the web link(s), the administrators of the sites posted an announcement to bring attention to the forthcoming survey and maximize participation. After the survey was made available, the administrators posted reminders at regular intervals to achieve maximum participation. The surveys were collected electronically on a password-protected account. Responses contained no identifiable personal information. Each response was linked to an Internet-provided address. Although we could not guarantee that an individual would not submit multiple responses, there were no Internet-provided addresses that appeared more than once in the collected responses.

The survey consisted of 13 questions (Table 1), and respondents who stated that they did not have a diagnosis of multiple hereditary exostoses were excluded. Respondents who did not answer whether they had been diagnosed with chondrosarcoma also were excluded. The survey was distributed in English only and could be completed in approximately 5 minutes, depending on the individual's command of the English language. 
Table 1. Survey distributed to each respondent via www.surveymonkey.com

(1) Do you have a diagnosis of multiple hereditary exostoses (MHE)? (yes or no)

(2) If you do NOT have a diagnosis of MHE, are you completing this survey on behalf of a relative (child, sibling, parent, grandparent, etc) who is diagnosed, or on behalf of a relative who was diagnosed but is now deceased? (yes or no)

(3) At what age were you or the patient diagnosed with MHE? (free text)

(4) To the best of your knowledge, have you or the patient had any surgeries related to MHE? (yes or no)

(5) If you answered yes to question 4, how many surgeries have you or the patient had? (free text)

(6) To the best of your knowledge, have you or the patient been diagnosed with chondrosarcoma as a result of your MHE? (yes or no)

(7) If yes, at what age were you or the patient diagnosed with chondrosarcoma? (free text)

(8) To the best of your knowledge, at which bone(s) were you or the patient diagnosed with chondrosarcoma? (free text)

(9) If you are completing this survey on behalf of someone else, what is your relationship to that patient (ie, child, parent, sibling, grandparent)? (free text)

(10) What is your current age (or that of the patient you are responding on behalf of)? (free text)

(11) Sex of the patient: (male or female)

(12) Country of birth: (free text)

(13) Please use the space below to add any comments, suggestions, or questions. Thank you for your participation. (free text)

All results were entered in a password-protected (and de-identified) Microsoft Excel (Redmond, WA, USA) database. Descriptive statistics (frequencies and means) were used to present results regarding patient demographics, status of multiple hereditary exostoses diagnosis, number of surgeries related to multiple hereditary exostoses diagnosis, status of chondrosarcoma diagnosis, and anatomic sites of chondrosarcoma lesions. No additional statistical analyses were performed.

We received survey responses from 793 individuals. Of the 793 respondents, 11 were excluded because they did not have a diagnosis of multiple hereditary exostoses. An additional 25 respondents were excluded because they did not answer whether they also had a diagnosis of chondrosarcoma. A total of 757 survey respondents qualified for our study. Of the submissions, 81 responses $(10.7 \%)$ were completed on behalf of an individual unable to complete the survey directly (eg, very young and elderly patients had surveys completed by their parents or caretakers).

\section{Results}

\section{Demographics of Patients with Multiple Hereditary} Exostoses

Females represented a slightly greater proportion of respondents, and age at diagnosis for male and female respondents was during the mid-first decade. Of the 742 respondents with a known diagnosis of multiple hereditary exostoses who reported their age, 56\% (419/742) were female and 44\% (323/742) were male. Fifteen respondents $(2 \% ; 15 / 757)$ did not list a sex. Median respondent age was 28 years (range, $<1-85$ years). Median ages were 25 years
Table 2. Demographic data of survey respondents

\begin{tabular}{llll}
\hline Parameter & $\begin{array}{l}\text { Total } \\
\text { respondents }\end{array}$ & $\begin{array}{l}\text { Male } \\
\text { respondents* }\end{array}$ & $\begin{array}{l}\text { Female } \\
\text { respondents* }\end{array}$ \\
\hline Number & 757 & $323(43 \%)$ & $419(55 \%)$ \\
$\begin{array}{l}\text { Median age (years) } \\
\begin{array}{l}\text { Mean age at diagnosis } \\
\text { of multiple hereditary } \\
\text { exostoses (years) }\end{array}\end{array}$ & $5.4( \pm 7.2)$ & $5.1( \pm 6.9)$ & $5.5( \pm 7.5)$ \\
\hline
\end{tabular}

* 15 respondents did not specify gender.

(range, $<1-85$ years) for males and 29 years (range, $<1-$ 82 years) for females. Thirty respondents (4\%) did not list an age (Table 2). Six hundred ninety-four respondents listed a country of birth from a total of 41 countries. Respondents from primarily English-speaking countries comprised the largest proportion who listed their country of birth, with the United States, the United Kingdom, Australia, and Canada making up $82.4 \%$ (572/694). Respondents from an additional 37 countries comprised the remaining $17.6 \%$ of surveys completed (Fig. 1).

\section{Surgical Procedures to Treat Multiple Hereditary Exostoses}

Survey results showed that patients with multiple hereditary exostoses often require surgery related to their diagnosis. Among the 590 respondents who answered whether they have required surgery, $418(70.8 \% ; 418 / 590)$ have had at least one surgery related to multiple hereditary exostoses, whereas 172 respondents $(29.2 \%, 172 / 590)$ have not. One hundred sixty-seven respondents did not answer whether they had surgery related to their diagnosis of 


\section{Geographic Distribution of Respondents}

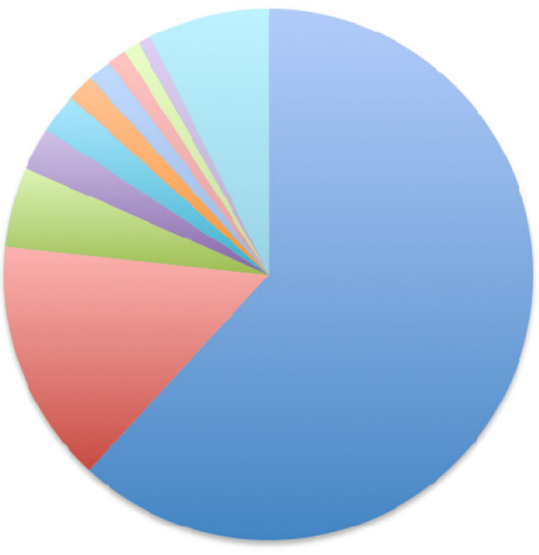

- United States, 379

United Kingdom, 91

Australia, 30

- Canada, 16

Norway, 15

New Zealand, 10

- Denmark, 9

Brazil, 7

Spain, 6

France, 5

Other, 45
Fig. 1 The geographic distribution of applicable respondents to our study survey is shown. Locations included Costa Rica (4), Israel (4), Portugal (4), Italy (3), Mexico (3), Sweden (3), Belgium (2), Germany (2), Guatemala (2), Indonesia (2), Jordan (2), Malta (2), Peru (2), Poland (2) Serbia (2), Algeria (1), Argentina (1), Bulgaria (1), Colombia (1), Finland (1), Haiti (1), India (1), Ireland (1), Japan (1), Netherlands (1), Panama (1), Puerto Rico (1), Romania (1), Russia (1), Singapore (1), South Africa (1), and South Korea (1).

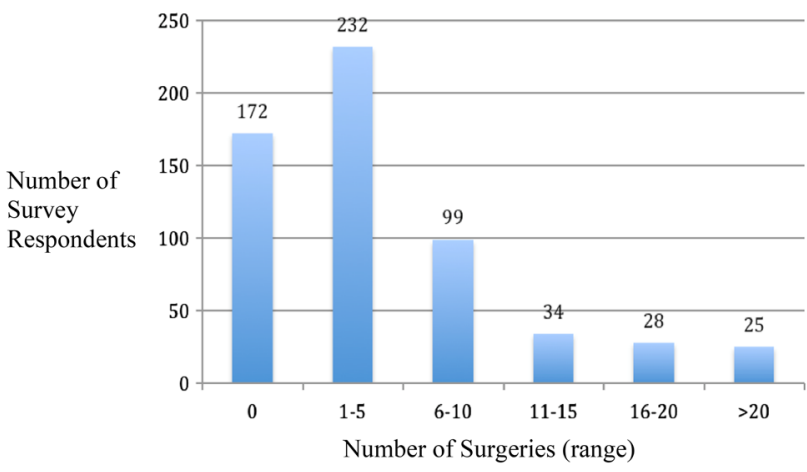

Fig. 2 The distribution of the number of surgical procedures respondents had as a representation of their disease burden is shown.

multiple hereditary exostoses. The majority $(96.6 \%$; 404/ 418) either has not required surgery or has had five or fewer procedures related to their multiple hereditary exostoses (Fig. 2). Patients with multiple hereditary exostoses without malignant change had a mean of $7.3( \pm 7.1)$ required surgeries in their lifetime. Patients with chondrosarcoma (Table 3$)$ had a mean of $8.8( \pm 9.4)$ lifetime procedures.

\section{Proportion With Malignant Degeneration}

The proportion of patients describing malignant degeneration was $2.7 \%$ ( 21 of 757 respondents), with the most common site being the pelvis (eight of 21 respondents),
Table 3. Demographic information and number of procedures

\begin{tabular}{|c|c|c|}
\hline Parameter & $\begin{array}{l}\text { Multiple } \\
\text { hereditary } \\
\text { exostoses }\end{array}$ & Chondrosarcoma \\
\hline Number & 757 & 21 \\
\hline Male* & $323(44 \%)$ & $8(38 \%)$ \\
\hline $\begin{array}{l}\text { Median age } \\
\text { (years)** }\end{array}$ & $28(<1-85)$ & $44(25-77)$ \\
\hline $\begin{array}{l}\text { Mean age at } \\
\text { diagnosis } \mathrm{N} \\
\text { (years) }\end{array}$ & $5.4( \pm 7.2)$ & $\begin{array}{l}7.6 \text { (multiple hereditary } \\
\text { exostoses, } \pm 5.5 \text { ) } 28.6 \\
(\text { chondrosarcoma, } \pm 9.3 \text { ) }\end{array}$ \\
\hline $\begin{array}{l}\text { Mean number of } \\
\text { procedures }\end{array}$ & $7.3( \pm 7.1)$ & $8.8( \pm 9.4)$ \\
\hline
\end{tabular}

followed by the scapula (four of 21 respondents). Of the 21 respondents with a diagnosis of chondrosarcoma, 13 were female and eight were male. They were diagnosed with multiple hereditary exostoses at a mean age of 7.6 years ( \pm 5.5 years), and with chondrosarcoma at a mean of 28.6 years $( \pm 9.3$ years, 25.8 years for females, 30 years for males; Table 3). Other anatomic sites of malignant degeneration included the proximal femur (three), rib (two), and clavicle, tibia, foot, and base of skull (Fig. 3). Of the 21 respondents with chondrosarcoma, two had local recurrence. A summary of the 21 respondents who reported malignant change including demographic information and details regarding the patients' chondrosarcoma diagnosis is provided (Table 4).

\section{Discussion}

In this study we sought to determine a more accurate proportion of patients with multiple hereditary exostoses who have had malignant degeneration. Earlier studies have been limited to narrowed geographic areas, and thus were not representative of the population at large. In using webbased resources dedicated to education and treatment of multiple hereditary exostoses, we sought to increase the catchment area that may have been unavailable to previous researchers of this topic. These platforms allowed for recruitment of a truly global cohort, thereby perhaps improving our ability to answer the posed question. Perhaps the most important complication of the benign lesions that are the hallmark of multiple hereditary exostoses is their malignant transformation to chondrosarcoma. Numerous studies have reported proportions from less than $1 \%$ to $25 \%$ for malignant degeneration of a benign exostosis to chondrosarcoma in patients who have multiple hereditary exostoses [3, 4, 7-9, 11-14]. In general, malignant transformation is rare and the true incidence has 


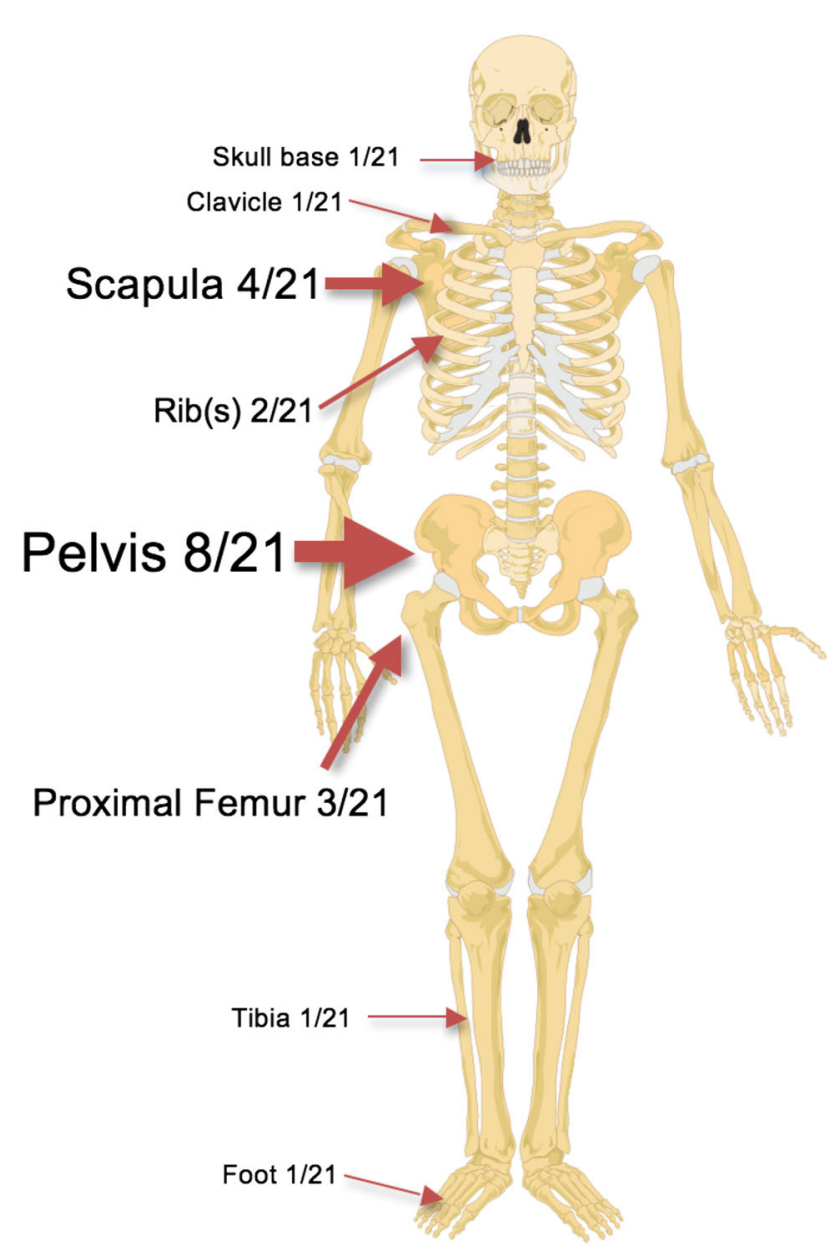

Fig. 3 The anatomic sites of the chondrosarcoma lesions among the 21 respondents who had malignant transformation are shown.

not been established [10]. This perhaps is because previous studies looked at cohorts from confined population clusters, or otherwise were subject to selection bias. In the current study, we attempted to further assess the question of risk in a larger, more varied, international population. We therefore aimed to describe the demographics of patients with multiple hereditary exostoses, assess the need for surgery as a rudimentary marker of disease burden, and elucidate the proportion of malignant transformation.

There are several limitations to our study. One is the inability to assess the overall response rate of the survey. The exact number of unique visitors to the various web sites on which our survey was posted is unknown. We have an estimate of the number of unique visitors to the web sites used, but this estimate cannot be used reliably to determine a true response rate. This lack of an accurate response rate is a limitation that is unavoidable in the study design. Our confidence in the data would be hampered more if the response rate were $0.5 \%$ compared with $50 \%$. Beyond the overall response rate, not all surveys were answered to completion. Respondents who did not answer whether they had a diagnosis of multiple hereditary exostoses and/or of chondrosarcoma were excluded. We believe these were the most important queries needed to answer our question. We included data from many incompletely answered surveys, which is a limitation. Furthermore, because the data were obtained via anonymous Internet response, there is no possibility of patient followup. Additionally, our survey was distributed only in English, which may have prevented some international community members from completing the survey. Recall bias also could be present, as individuals may not have a good understanding of what chondrosarcoma and multiple hereditary exostoses are. Examples of recall bias may be the self-reported sites of chondrosarcoma. The survey asked respondents to respond "to the best of your knowledge." This allows for inherent recall bias. However, we believe this point is mitigated somewhat by respondents either having logged on to or browsed these web-based support groups and social media sites dedicated specifically to multiple hereditary exostoses. Thus, most respondents might logically have a basic knowledge of their condition, to include specific anatomic sites of malignant change. Some individuals $(10.7 \%)$ had the survey completed on their behalf, which seemingly would introduce additional recall bias. Perhaps the most important limitation of this study is recruitment and selection bias predispose for the survey to be completed by those who have more serious sequelae of multiple hereditary exostoses. Such people logically would be more apt to seek out web-based support groups such as those used in our study and would be more likely to participate. Patients with less severe sequelae of multiple hereditary exostoses either may be less likely to participate or may not know they have multiple hereditary exostoses. Finally, and perhaps equally important is that the survey collected responses from all ages. This would limit the utility of the data if, for instance, a 5-year-old patient is measured equally against a 75 -year-old patient. The older patient undoubtedly would have required more treatment during his or her lifetime and had the time for more symptomatic lesions to develop. Differences in age among respondents also may have resulted in additional bias in that the nature of the web-based survey may trend toward more accurate responses among younger, more computer-savvy respondents. It stands to reason that respondents from younger generations would be more familiar with the web-based platforms and social media sites. We believe our survey results reflect a cross-section of a heterogeneous population with multiple hereditary exostoses.

The age and sex of our study population were similar to those of two of the larger (several hundred participants) studies. Pedrini et al. [11] had a study population that consisted of $50.7 \%$ males and $49.3 \%$ females while Goud 
Table 4. Demographic and descriptive data for patients with chondrosarcoma

\begin{tabular}{|c|c|c|c|c|c|}
\hline $\begin{array}{l}\text { Age } \\
\text { (years) }\end{array}$ & Sex & Country of birth & $\begin{array}{l}\text { Age at diagnosis of } \\
\text { multiple hereditary } \\
\text { exostoses (years) }\end{array}$ & $\begin{array}{l}\text { Age at diagnosis } \\
\text { of chondrosarcoma } \\
\text { (years) }\end{array}$ & Site of chondrosarcoma \\
\hline 28 & $\mathrm{~F}$ & USA & 22 & 22 & $5^{\text {th }} \mathrm{rib}$ \\
\hline 67 & $\mathrm{~F}$ & USA & 14 & 22 & $\begin{array}{l}\text { Pubic ramus and } \\
\text { ischium }\end{array}$ \\
\hline 36 & $\mathrm{~F}$ & Norway & 5 & 34 & Lesser trochanter \\
\hline 57 & M & USA & 9 & 48 & Right femur \\
\hline 77 & F & USA & 6 & 19 & Left clavicle \\
\hline 55 & $\mathrm{~F}$ & USA & 10 & 14 & Right scapula \\
\hline 57 & $\mathrm{~F}$ & USA & 3 & $37,45^{*}$ & Sternum, scapula \\
\hline 29 & M & Norway & $<1$ & 25 & Proximal femur \\
\hline 29 & M & Norway & 1 & 26 & Hip \\
\hline 40 & $\mathrm{~F}$ & USA & 5 & 25 & Left iliac wing \\
\hline 45 & $\mathrm{~F}$ & UK & 13 & 37 & Scapula \\
\hline 27 & $\mathrm{~F}$ & USA & 7 & 25 & Right $9^{\text {th }}$ rib \\
\hline 25 & $\mathrm{~F}$ & Scotland, UK & $<1$ & 21 & Pelvis \\
\hline 67 & $\mathrm{~F}$ & USA & 13 & 19 & $\begin{array}{l}\text { Right pubic ramus } \\
\text { and ischium }\end{array}$ \\
\hline 39 & M & UK & 12 & 33 & Foot \\
\hline 47 & M & Belgium & 1 & 45 & Pelvis \\
\hline 32 & $\mathrm{~F}$ & USA & 11 & 31 & Pubic ramus \\
\hline 43 & M & USA & 5 & 20 & Scapula \\
\hline 35 & $\mathrm{~F}$ & USA & 7 & 30 & Tibia \\
\hline 47 & M & Belgium & 10 & 43 & Pelvis \\
\hline 42 & $\mathrm{~F}$ & UK & 5 & $25,39^{*}$ & Left hip/pelvis \\
\hline
\end{tabular}

* Chondrosarcoma recurrence.

et al. [4] had a study population of $45 \%$ males and 55\% females [4, 11]. Our study population included 56\% females and $44 \%$ males. Pedrini et al. [11] did not specify the age of their cohort but proposed a classification scheme based on skeletal deformity and functional limitation that included patients in childhood, preadolescence, adolescence, and skeletal maturity. Goud et al. [4] reported a cohort with a median age of 29.1 years (range, 1-80 years) that closely mirrors the median age of respondents in our study, which is 28.7 years (range, $<1-85$ years). Thus we believe our survey population is a worthy cross section of patients with multiple hereditary exostoses.

Goud et al. [4] reported that beyond the effects of pain, multiple hereditary exostoses often causes limitations in patient employment, physical activities (especially sports), and activities of daily living, thereby more comprehensively quantifying the burden of disease. Goud et al. [4] reported that $88 \%$ of adults and $48 \%$ of children in their study population required at least one surgical procedure to excise an osteochondroma. They also noted that $22 \%$ of their cohort required deformity correction. In a cohort of 143 patients, Schmale et al. [13] noted that $74 \%$ required surgery to remove at least one osteochondroma and the average patient required three surgical procedures related to their diagnosis of multiple hereditary exostoses. Our results showed that, of respondents who answered whether they have had surgery, $70.8 \%$ (418/590) have had at least one surgical procedure. The mean number of surgeries related to multiple hereditary exostoses was 7.3 ( \pm 7.1 surgeries). This higher number of surgeries, when compared with the number reported by Schmale et al. [13], may be attributable to the selection and recruitment bias toward patients with more severe disease. Patients with more serious disease would likely be more apt to use or visit web-based support groups and social media sites dedicated to multiple hereditary exostoses.

The proportion of patients who had malignant change $(2.7 \%)$ is within a few percentage points of the proportions reported in previous studies [3, 4, 8, 9, 11]. Pedrini et al. [11] reported 5\% with malignant change, whereas Goud et al. reported 7\% [4]. Although we believe an important bias in our study may have resulted from a trend in participation by patients with more burdensome disease, the proportion of malignant change in our respondents was less than in the studies mentioned. This perhaps is because our survey looked at a larger, more heterogeneous population that weighed patients of all ages equally. Patients from our cohort 
who had malignant transformation did so at a mean age of 28.6 years ( \pm 9.3 years). This is more than 6 years earlier than the cohort of Goud et al. [4], who experienced malignant transformation at a mean age of 35 years [4]. Smaller cohorts in previous studies had mean ages at the time of malignant change of 31 and 39 years [7,9]. The younger age of patients in our cohort at the time of change may be attributable to the aforementioned bias toward patients with more serious disease to complete the survey. Three survey respondents from our cohort reported malignant change in their second decade, with the youngest at 14 years. This underscores the importance for clinicians to be cognizant of the risk for malignant change as early as the adolescent years. In our respondents, malignant degeneration of lesions occurred most often in the pelvis (eight of 21) and scapula (four of 21), a distribution similar to those reported previous studies that noted location of chondrosarcoma lesions $[11,13]$. This may be attributable to a delayed diagnosis of lesions in these areas [1]. Nevertheless, the risk of chondrosarcoma in patients with multiple hereditary exostoses is low, albeit greater than the risk to the general population. The relatively low incidence of chondrosarcoma in some families and apparent greater incidence in others may reflect variable penetrance of disease, chance occurrence, environmental effects, epistasis, or some combination of these, and likely, other factors [3].

In a large, heterogeneous cohort of patients diagnosed with multiple hereditary exostoses we found that the mean age of patients at the time of diagnosis of multiple hereditary exostoses is 5.4 years ( \pm 7.2 years), that patients have a mean of 7.3 surgeries ( \pm 7.1 surgeries), and that the proportion of survey respondents who reported malignant transformation was $2.7 \%$ at a mean age of 28.6 years ( \pm 9.3 years). In discussing the sequelae of multiple hereditary exostoses, clinicians perhaps might use this study to offer an unspecific statement of risk of malignant degeneration of this disorder among the population at large. Further studies that quantify the burden of multiple hereditary exostoses in a larger, more-diverse population might help show the true cost of the disease to the individual patient and society. Multiple hereditary exostoses is a chronic ailment with the risk of malignant transformation; patients with this diagnosis should be monitored regularly. Social media and other web-based platforms may play an important role in psychosocial support for patients with a diagnosis of multiple hereditary exostoses, local and regional referral networks, and important epidemiologic data to advance our understanding of the disorder.

Acknowledgments We thank Michela Schillinger for assistance with the background research and statistical analysis of this study.

\section{References}

1. Altay M, Bayrakci K, Yildiz Y, Erekul S, Saglik Y. Secondary chondrosarcoma in cartilage bone tumors: report of 32 patients. J Orthop Sci. 2007;12:415-423.

2. Cook A, Raskind W, Blanton SH, Pauli RM, Gregg RG, Francomano CA, Puffenberger E, Conrad EU, Schmale G, Schellenberg G, Wijsman E, Hecht JT, Wells D, Wagner MJ. Genetic heterogeneity in families with hereditary multiple exostoses. Am J Hum Genet. 1993;53:71-79.

3. Gordon SL, Buchanan JR, Ladda RL. Hereditary multiple exostoses: report of a kindred. J Med Genet. 1981;18:428-430.

4. Goud AL, de Lange J, Scholtes VA, Bulstra SK, Hams SJ. Pain, physical and social functioning, and quality of life in individuals with multiple hereditary exostoses in the Netherlands: a national cohort study. J Bone Joint Surg Am. 2012;94:1013-1020.

5. Hattori H, Asagai Y, Yamamoto K. Sudden onset of saphenous neuropathy associated with hereditary multiple exostoses. J Orthop Sci. 2006;11:405-408.

6. Hennekam RC. Hereditary multiple exostoses. J Med Genet. 1991;28:262-266.

7. Kivioja A, Ervasti H, Kinnunen J, Kaitila I, Wolf M, Böhling T. Chondrosarcoma in a family with multiple hereditary exostoses. J Bone Joint Surg Br. 2000;82:261-266.

8. Legeai-Mallet L, Munnich A, Maroteaux P, Le Merrer M. Incomplete penetrance and expressivity skewing in hereditary multiple exostoses. Clin Genet. 1997;52:12-16.

9. Luckert-Wicklund C, Pauli RM, Johnston D, Hecht JT. Natural history study of hereditary multiple exostoses. Am J Med Genet. 1995;55:43-46.

10. Mavrogenis AF, Angelini A, Drago G, Merlino B, Ruggieri P. Survival analysis of patients with chondrosarcomas of the pelvis. J Surg Oncol. 2013;108:19-27.

11. Pedrini E, Jennes I, Tremosini M, Milanesi A, Mordenti M, Parra A, Sgariglia F, Zuntini M, Campanacci L, Fabbri N, Pignotti E, Wuyts W, Sangiorgi L. Genotype-phenotype correlation study in 529 patients with multiple hereditary exostoses: identification of "protective" and "risk" factors. J Bone Joint Surg Am. 2011;93: 2294-2302.

12. Porter DE, Lonie L, Fraser M, Dobson-Stone C, Porter JR, Monaco AP, Simpson AH. Severity of disease and risk of malignant change in hereditary multiple exostosis: a genotypephenotype study. J Bone Joint Surg Br. 2004:86:1041-1046.

13. Schmale GA, Conrad EU $3^{\text {rd }}$, Raskind WH. The natural history of hereditary multiple exostoses. J Bone Joint Surg Am. 1994; 76:986-992.

14. Solomon L. Hereditary multiple exostosis. J Bone Joint Surg Br. 1963:45:292-304.

15. Wu YQ, Heutlink P, de Vries BB, LA, van den Ouweland AM, Niermeijer MF, Galjaard H, Reyniers E, Willems PJ, Halley DJ. Assignment of a second locus for multiple exostoses to the pericentromeric region of chromosome 11. Hum Mol Genet. 1994;3: $167-171$

16. Wuyts W, Ramlakhan S, Van Hul W, Hecht JT, van den Ouweland AM, Raskind WH, Hofstede FC, Reyniers E, Wells DE, de Vries B, Conrad EU, Hill A, Zalatayev D, Weissenbach J, Wagner MJ, Bakker E, Halley DJ, Willems PJ. Refinement of the multiple exostoses locus (EXT2) to a 3-cM interval on chromosome 11. Am J Hum Genet. 1995;57:382-387. 